\title{
Extended Spectrum Beta-lactamase Producing Gram Negative Bacterial Isolates from Urine of Patients Visiting Everest Hospital, Kathmandu, Nepal
}

\author{
Nisha Guragain ${ }^{1}$, Aashish Pradhann ${ }^{2}$, Binod Dhungel ${ }^{1}$, Megha Raj Banjara' ${ }^{1}$, Komal Raj Rijal ${ }^{1 *}$, \\ Prakash Ghimire ${ }^{1}$ \\ ${ }^{1}$ Central Department of Microbiology, Tribhuvan University, Kathmandu, Nepal \\ ${ }^{2}$ Everest Hospital, Baneshwor, Kathmandu
}

*Corresponding author: Dr. Komal Raj Rijal, Associate Professor, Central Department of Microbiology, Tribhuvan University, Kirtipur, Kathmandu, Email: rijalkomal@gmail.com

\begin{abstract}
Objectives: The study was aimed to determine the prevalence of Extended Spectrum Beta Lactamase (ESBL) producing Gram negative pathogens from urine samples along with their antimicrobial resistance.

Methods: This cross-sectional study was conducted from December 2015 to May 2016 at Everest Hospital, Kathmandu. Mid-stream urine samples were collected and processed for culture by standard loop streak method. Identified bacterial isolates were tested for Antibiotic Susceptibility by modified Kirby Bauer disc diffusion method and, were subjected to ESBL screening by using $30 \mu \mathrm{g}$ cefotaxime and ceftazidime. ESBL production was confirmed by combination disc method.
\end{abstract}

Results: Of the three hundred urine samples, 22.7\% (67/300) showed significant growth. Four different bacterial species were identified. Among the isolates, E. coli was the most common pathogen (71.64\%) followed by Klebsiella pneumoniae (14.92\%), Pseudomonas spp (8.95\%) and Acinetobacter spp $(4.48 \%)$. Altogether $92.54 \%(n=62)$ isolates were sensitive to gentamicin, $89.55 \%(n=60)$ to amikacin, and $79.10 \%(n=53)$ to nitrofurantoin. $70.10 \%(n=47)$ isolates were resistant to antibiotic ampicillin while $62.68 \%(n=42)$ were found as multi-drug resistant (MDR) and 29.8\% ( $n=20)$ were ESBL producers.

Conclusion: The overall prevalence of MDR and ESBL among uropathogens is low in comparison to other studies though it is essential to have a regular monitoring of ESBL producing clinical isolates in laboratory practice.

Key words: Uropathogens, Mid-stream urine, Antimicrobial resistance, ESBL, MDR

\section{INTRODUCTION}

Urinary tract infection (UTI) is a common bacterial infection prevailing in developing countries like Nepal. UTI is defined as a condition in which the urinary tract is infected with a pathogen causing inflammation. The emergence and occurrence of UTI is increasing day by day. The major Gram-negative bacteria involved in causing UTIs are E. coli, Klebsiella spp, Proteus spp, Pseudomonas spp, Citrobacter spp, Acinetobacter spp with most leading uropathogens E. coli and Klebsiella pneumoniae that belongs to Enterobacteriaceae family (Dromigney et al. 2005).

Clinical experience has indicated the presence of numerous cases of antibiotic resistance to common antibiotics by uropathogens in both developed and developing countries (Gupta 2002). The resistivity has posed challenges in choosing empiric regimens. The cause for resistivity against most prescribed broadspectrum beta-lactam antibiotic for treatment against
Date of Submission: October 16, 2019

Published Online: December, 2019
Date of Acceptance: November 15, 2019

DOI: https:/ / doi.org/10.3126/tujm.v6i0.26575 
Gram negative bacterial infection is the production of extended spectrum beta lactamases. These ESBLs enable these bacilli highly efficient in inactivating third generation cephalosporins, monobactams and penicillins (Hawkey 2008) but cannot inactivate cephamycins or carbapenems and are inhibited by clavulanic acid (Bradford 2001; Bush 2001). Several risk factors for ESBL producing Gram negative bacterial infections have been described for the most frequent antimicrobial exposure mostly to third generation cephalosporins resulting in increased morbidity, mortality and costs of health care (Chakraborty et al. 2016).

Prevalence of ESBL producing uropathogens varies widely even in closely related regions. Various studies have reported ESBL producing bacteria in Nepal. Failure in the treatment of infection especially caused by ESBL producing organisms need to be under controlled monitoring in developing countries to avoid widespread distribution of multidrug resistant uropathogens (Chakrawarty et al. 2015). Therefore, this study seeks to evaluate the prevalence of ESBL producing Gram negative bacterial isolates and their existing antibiotic susceptibility pattern.

\section{MATERIALS AND METHODS}

This cross-sectional hospital based prospective study was conducted from December 2015-May 2016 in microbiological laboratory of Everest Hospital. Patients (inpatients and outpatients) clinically suspected of UTI of different ages and sexes were selected for the study. A total of 300 mid-stream urine samples were collected and processed according to standard operating protocols during the study period.

Suspected samples were cultured onto Cystine Lactose Electrolyte Deficient (CLED) agar (Hi-Media Pvt. Ltd., India) and Gram-negative bacteria were isolated. The isolates with significant bacteriuria of $10^{5}$ colonies $/ \mathrm{ml}$ were identified based upon the standard laboratory procedures involving morphological characteristics, Gram's staining, rapid tests(catalase and oxidase) and biochemical tests IMViC (Indole, Methyl red, Voges Proskauer, Citrate), triple sugar iron agar test, oxidation-fermentation test and urease test (Harley and
Prescott 2002). Each identified isolate was subjected to invitro antibiotic susceptibility test by modified Kirby-Bauer disc diffusion method as recommended by CLSI guidelines on Muller Hinton Agar (CLSI 2006). Commercially available antibiotic tested were ampicillin, amikacin, cotrimoxazole, ofloxacin, nitrofurantoin, nalidixic acid, ceftriaxone, gentamicin and imipenem. MDR isolates were detected based on their resistance to two or more antibiotics (Cheesbrough 2006; CLSI 2014).

The isolates exhibiting reduced susceptibility to cefotaxime $(30 \mu \mathrm{g})$ and ceftazidime $(30 \mu \mathrm{g})$ were considered as potential ESBL producers. The ESBL production was phenotypically confirmed by combination disc method (CLSI 2014). The disc used were cefotaxime and ceftazidime alone and cefotaxime and ceftazidime in combination with clavulanic acid. $A \geq 5 \mathrm{~mm}$ increase in growth inhibition zone for any antimicrobial associated with clavulanic acid in comparison with the inhibition zone of antibiotic tested alone confirmed ESBL production.

The collected data analysis was done by SPSS version 20 software and chi-square test was done as a test of significance.

\section{RESULTS}

Out of 300 mid-stream urine samples collected, 22.3\% ( $n=67)$ showed the significant growth among 12 inpatients and 288 outpatients of different age groups and of both the sexes. Four different Gram negative bacteria were isolated; Escherichia coli, Klebsiella pneumoniae, Pseudomonas aeruginosa and Acinetobacter spp.

From the samples collected between 0-80 age groups, the maximum growth was observed in age group 20-30 years i.e. 22 (32.8\%) followed by age group 3040 years $9(13.4 \%)$ and $70-80$ years $9(13.4 \%)$. Among 119 male, $21(17.64 \%)$ showed significant growth and among female 46 (25.4\%) showed significant growth. Among males, maximum growth was observed in 2030 years $(23.8 \%)$ whereas in females maximum number of growth was found in 20-30 years (37\%) followed by age group $10-20$ years (15.21\%). The prevalence of UTI was found higher in female than male (Table 1). 
Guragain et al. 2019, TUJM 6(1): 26-31

Table 1: Age and gender wise distribution of patients with isolates

\begin{tabular}{|c|c|c|c|c|c|c|}
\hline \multirow{2}{*}{ Age group } & \multicolumn{2}{|c|}{ Male } & \multicolumn{2}{|c|}{ Female } & \multicolumn{2}{|c|}{ Total } \\
\hline & No. & $\%$ & No. & $\%$ & No. & $\%$ \\
\hline $0-10$ & 3 & 14.28 & 3 & 6.5 & 6 & 8.96 \\
\hline $10-20$ & 1 & 4.76 & 7 & 15.21 & 8 & 11.94 \\
\hline $20-30$ & 5 & 23.8 & 17 & 36.95 & 22 & 32.83 \\
\hline $30-40$ & 4 & 19.04 & 5 & 10.86 & 9 & 13.43 \\
\hline $40-50$ & 1 & 4.76 & 5 & 10.86 & 6 & 8.96 \\
\hline $50-60$ & 1 & 4.76 & 3 & 6.5 & 4 & 5.97 \\
\hline $60-70$ & 1 & 4.76 & 2 & 4.34 & 3 & 4.48 \\
\hline $70-80$ & 5 & 23.8 & 4 & 8.69 & 9 & 13.43 \\
\hline Total & 21 & & 46 & & 67 & \\
\hline
\end{tabular}

Similarly, from 300 mid-stream urine samples collected, only four different Gram negative bacteria were isolated. Among them, Escherichia coli was

found predominant with significant growth of $71.64 \%$ followed by Klebsiella pneumoniae (14.93\%), Pseudomonas aeruginosa (8.95\%) and Acinetobacter (4.5\%) (Table 2)

Table 2: Microbiological profile of urine isolates

\begin{tabular}{lcc}
\hline \multicolumn{1}{c}{ Organism isolated } & Total no. of isolates & $\%$ \\
\hline Escherichia coli & 48 & 71.64 \\
Klebsiella pneumoniae & 10 & 14.93 \\
Pseudomonas aeruginosa & 6 & 8.95 \\
Acinetobacter spp & 3 & 4.48 \\
\hline \multicolumn{1}{c}{ Total } & 67 & 100 \\
\hline
\end{tabular}

The antibiotic susceptibility test profile of the identified isolates was determined by modified Kirby Bauer disc diffusion method. The Gram-negative bacteria showed highest sensitivity to gentamicin (92.54\%), amikacin
$(89.55 \%)$ and nitrofurantoin $(79.10 \%)$ respectively. Similarly high resistant rate was found against ampicillin (70.15\%), nalidixic acid (46.26\%) followed by cotrimoxazole and ceftriaxone (32.83\%). (Table 3)

Table 3: Antibiotic susceptibility profile of Gram-negative isolates

\begin{tabular}{lccccccc}
\hline \multirow{2}{*}{ Antibiotics } & \multicolumn{2}{c}{ Sensitive } & \multicolumn{2}{c}{ Intermediate } & \multicolumn{2}{c}{ Resistant } & Total \\
\cline { 2 - 8 } & No. & $\%$ & No. & $\%$ & No. & $\%$ & \\
\hline Ampicillin & 18 & 26.8 & 2 & 2.985 & 47 & 70.15 & 67 \\
Ofloxacin & 47 & 70.15 & 4 & 5.97 & 16 & 23.88 & 67 \\
Nalidixic acid & 30 & 44.77 & 6 & 8.95 & 31 & 46.26 & 67 \\
Nitrofurantoin & 53 & 79.10 & 6 & 8.95 & 8 & 11.94 & 67 \\
Cotrimoxazole & 36 & 53.73 & 9 & 8.95 & 22 & 32.83 & 67 \\
Ceftriaxone & 38 & 56.72 & 7 & 10.45 & 22 & 32.83 & 67 \\
Amikacin & 60 & 89.55 & 4 & 5.97 & 3 & 4.48 & 67 \\
Imipenem & 37 & 55.22 & 4 & 5.97 & 26 & 38.80 & 67 \\
Gentamicin & 62 & 92.54 & 1 & 1.49 & 4 & 5.97 & 67 \\
\hline
\end{tabular}

About $62.68 \%$ of total Gram-negative isolates were MDR. Higher rate of MDR was observed in E. coli $(66.7 \%)$. Similarly, out of 67 isolates, 20 were confirmed as ESBL producer. Prevalence of ESBL was found high in E. coli $31.25 \%(15 / 48)$. Total prevalence of ESBL producing Gram negative bacteria was $29.8 \%$. There was no significant association between Gram negative isolates and ESBL production $(\mathrm{p} \leq 0.05)$ (Table 4). 
Table 4: Profile of ESBL producing bacterial isolates

\begin{tabular}{lccc}
\hline \multicolumn{1}{c}{ Organism isolated } & Total no. of isolates & No. of MDR (\%) & ESBL producers (\%) \\
\hline Escherichia coli & 48 & $32(66.7)$ & $15(31.25)$ \\
Klebsiella spp & 10 & $4(40)$ & $3(30)$ \\
Pseudomonas spp & 6 & $4(66.7)$ & $1(16.7)$ \\
Acinetobacter & 3 & $2(66.7)$ & $1(33.3)$ \\
\hline \multicolumn{1}{c}{ Total } & 67 & 42 & $20(29.8)$ \\
\hline
\end{tabular}

\section{DISCUSSION}

Increasing number of recent reports on bacterial resistance to beta-lactam antibiotics is of serious concern today as these drugs are used for treatment of most bacterial infections. Failure of empirical therapy is increasing proportionally to increasing rate of ESBL producing pathogens. So, detection of ESBL producing bacteria is highly important and this study was carried out with the same motive.

In this study, out of 300 midstream urine samples processed, $22.7 \%(n=67)$ showed the significant growth. This growth rate is similar to the other study done in Nepal which have shown $16.88 \%$ and $16 \%$ growth rate (Poudyal et al. 2011; Tiwari 2014). This growth rate was found higher than the study done by Chander and Shrestha, 2013 with 9.34\% whereas lower than the study of Karki 2010 with $58.8 \%$. The study showed higher prevalence of UTI among females $68.66 \%(n=46)$ than in males $31.43 \%(n=21)$. This rate is similar to study done by Yadav and Satyam 2017; Chaudhary et al. 2016. The reason for higher rate of UTI in female is due to their shorter length of urethra and complex physiology. In female higher growth rate was found in the age group of $20-30$ years of age $36.95 \%$ which may be due to their sexual activity during this period-a potential factor for UTI. Also, at the age group of 1020 years, growth was found higher. This might be due to their poor sanitary practices and hormonal changes during the phase.

In this study, out of 67 bacteria isolated, maximum number of $E$. coli was isolated with $71.64 \% \quad(n=48)$ followed by Klebsiella pneumoniae 14.93\% ( $\mathrm{n}=10)$, Pseudomonas aeruginosa $8.95 \%(\mathrm{n}=6)$ and Acinetobacter $4.48 \%(n=3)$ respectively. Similar predominance of $E$. coli was found in the recent study which showed $84 \%$ growth of E. coli and 16\% of Klebsiella (Yadav et al. 2015). Likewise, similar results were seen in other studies done by Baral et al. 2012; Das et al. 2000; Sharma et al. 2000. The reason for the higher isolation of $E$. coli is due to their commensalism property with ability to bind to the glycoconjugate receptor of epithelial cells of human urinary tract. Klebsiella is another major uropathogens isolated from urine samples. These bacteria have several defense mechanisms enabling them to spread infection faster.

In this study, gentamicin and amikacin with the susceptibility rate of $92.54 \%$ and $89.55 \%$ respectively were found to be the most active drug against Gram negative isolates followed by nitrofurantoin with $79.10 \%$ susceptibility. Ampicillin was found as the most resistant drug with $70.10 \%$ followed by other antibiotics: ofloxacin, nalidixic acid, cotrimoxazole and ceftriaxone.

In this study, $62.68 \%$ of the total Gram negative isolates were found as MDR. This result was in consistent with the study reported from National Public Health Laboratory (Poudyal et al. 2011). In our study, ESBL production rate was $33.3 \%, 31.25 \%, 30 \%$ and $16.7 \%$ of Acinetobacter, E. coli, Klebsiella pneumoniae and Pseudomonas aeruginosa respectively. The overall prevalence of ESBL production was $29.8 \%$. This rate is slightly lower in compare to other studies from Nepal. Though previous studies have shown similar prevalence of $24 \%, 25 \%, 25.8 \%, 26.8 \%, 31.3 \%$ and $33.2 \%$ (Ansari et al. 2015, Khanal et al. 2013, Neupane et al. 2016; Pant et al. 2016; Pokharel et al. 2014; Yadav et al. 2015) whereas higher than the study of Chander and Shrestha 2013 which have reported $13.5 \%$. The cause for lower prevalence rate of ESBL producing uropathogens may be due to low sample collection and low patient flow in the hospital. ESBL prevalence is increasing day by day due to self-medication, suboptimal quality of antimicrobial drugs and poor community and personal hygiene (Walson et al. 2001).

\section{CONCLUSION}

E. coli was yet again the predominant bacteria isolated from urine sample. Gram negative isolates were highly sensitive to gentamicin, amikacin followed by nitrofurantoin and highly resistant to ampicillin. 


\section{ACKNOWLEDGEMENTS}

We would like to express our sincere gratitude and admiration to all the staffs and faculties of Central Department of Microbiology, Tribhuvan University, Kirtipur and Everest Hospital, Kathmandu for their support and guidance to complete this study.

\section{CONFLICT OF INTEREST}

The authors declare that they have no competing interests.

\section{REFERENCES}

Ansari S, Nepal HP, Gautam R, Shrestha S, Neupane P, Gurung G and Chapagain ML (2015). Community acquired multi-drug resistant clinical isolates $E$. coli in a tertiary care center of Nepal. Antimicrob Resist and Infect Control 4: 15.

Baral P, Neupane S, Marasini BP, Ghimire KR, Lekhak B and Shrestha B (2012). High prevalence of multidrug resistance in bacterial pathogens from Kathmandu, Nepal. BMC Research Notes 5: 38.

Bradford PA (2001). Extended spectrum betalactamases in the $21^{\text {st }}$ century: Characterization, epidemiology and detection of this important resistance threat. Clin Microbiol Rev 14(4): 933-951.

Bush K (2001). New beta-lactamases in Gram negative bacteria: diversity and impact on the selection of antimicrobial therapy. Clin Infect Dis 3(7): 10851089.

Chakraborty S, Mahsna K, Sarker PK, Alam MDZ, Karin MIA and Sayem SMA (2016). Prevalence, antibiotic susceptibility profiles and ESBL production in Klebsiella pneumoniae and Klebsiella oxytoca among hospitalized patients. Peridicum Biologorum 118 (1): 53-58.

Chakrawarty A, Dongol P, Khanal H, Subba P and Banerjee JJ (2015). Extended spectrum betalactamases detection and multiple antibiotic existance indexing of E. coli from urine samples of patients from a referral hospital of Eastern Nepal. Int J Appl Sci Biotechnol 3(3): 423-426.

Chander and Shrestha (2013). Prevalence of extended spectrum beta-lactamase producing Escherichia coli and Klebsiella pneumoniae urinary isolates in a tertiary care hospital in Kathmandu, Nepal. BMC Research Notes 6: 487.

Chaudhary P, Bhandari D, Thapa K, Thapa P, Shrestha
D, Chaudhary HK, Shrestha A, Parajuli H and Gupta BP (2016). Prevalence of Extended spectrum beta-lactamase producing Klebsiella pneumoniae isolated from urinary tract infected patients. J Nepal Health Res Counc 14(33): 111-115.

Cheesbrough M (2006). District laboratory Practice in Tropical Countries Part-2, Cambridge University Press.

Clinical and Laboratory Standards Institute/ NCCLS (2006). Performance Standards for antimicrobial susceptibility testing; $15^{\text {th }}$ informational supplement. CLSI/NCCLS M100-S15: Clinical and Laboratory Standards Institute, Wayne PA.

Clinical and Laboratory Standards Institute/NCCLS (2014). Performance Standards for antimicrobial susceptibility testing; $15^{\text {th }}$ informational supplement. CLSI/NCCLS M100-S15. Clinical and Laboratory Standards Institute.

Dromigney JA, Nabeth P, Juergens-Behr A and Perrier Ggros-Claude JD (2005). Risk factors for antibiotic resistant Escherichia coli isolated from community acquired urinary tract infections in Dakar, Senegal. J Antimicrob Chemother 56: 236239.

Hawkey PM (2008). Prevalence and clonality of extended spectrum beta-lactamases in Asia. Clin Microbiol Infect 14 (Suppl 1): 159-65.

Karki M (2010). Prevalence of microorganisms in the intensive care unit (ICU) patients and their association with indoor environment. MSc dissertation submitted to the Central Department of Microbiology, Tribhuvan University. pp: 7-88

Kateregga JK, Kantume R, Atuhaire C, Lubowa MN and Ndukui JG (2015). Phenotypic expression and prevalence of ESBL producing Enterobacteriaceae in samples collected from patients in various wards of Mulago Hospital, Uganda. BMC Pharmacology and Toxicology 16:14.

Khanal S, Joshi DR, Bhatta DR, Devkota U and Pokhrel BM (2013). Beta-lactamase producing multidrug resistant bacterial pathogens from tracheal aspirates of intensive care unit patients at National Institute of Neurological and Allied Sciences, Nepal. ISRN Microbiol 2013: 847569.

Koirala J, Pokhrel BM, Dahal RK, Mishra SK, Khadka 
PK and Tuladhar NR (2006). Multi-drug resistant and extended spectrum beta lactamases (ESBL) producing Salmonella enterica (Serotypes Typhi and Paratyphi A) from blood isolates in Nepal: Surveillance of resistance and a search for newer alternatives. Int J Infect Dis 10: 434-438.

Neupane S, Pant ND, Khatiwada S, Chaudhary R and Banjara MR (2016). Correlation between biofilm formation and resistance towards different commonly used antibiotics along with ESBL production in uropathogenic Escherichia coli isolated from the patients suspected of urinary tract infection visiting Shree Birendra Hospital, Chhauni, Kathmandu, Nepal. Antimicrob Resist Infect Control 5 :5.

Panta K, Ghimire P, Rai SK, Mukhiya RK, Singh RN and Rai G (2013). Antibiogram typing of Gram negative isolates in different clinical samples of a tertiary hospital. Asian J Pharm Clin Res 6: 153-156.

Pant ND, Bhandari R, Poudel A and Sharma M (2016). Assessment of the effectiveness of three different cephalosporins/clavulanate combinations for the phenotypic confirmation of extended spectrum beta-lactamases producer bacterial isolates from urine samples at National Public Health Laboratory, Kathmandu, Nepal. BMC Research
Notes 9: 390

Pfaller MA and Segretti J (2006). Overview of the epidemiological profile and laboratory detection of extended spectrum beta-lactamases. Clin Infect Dis 15(42): 153-163.

Poudyal S, Bhatta DR, Shakya G, Upadhyaya B, Dumre SB and Buda G (2011). Extended spectrum betalactamases producing multidrug resistant clinical bacterial isolates at National Public Health Laboratory, Nepal. Nepal Med Coll J 13(1): 34-38.

Pokhrel RH, Thapa B, Kafle R, Shah PK and Tribuddharat C (2014). Co-existence of betalactamases in clinical isolates of Escherichia coli from Kathmandu, Nepal. BMC Research Notes 7:694

Walson JL, Marshall B, Pokhrel BM, Kafle KK and Levy SB (2001). Carriage of antibiotic-resistant faecal bacteria in Nepal reflects proximity to Kathmandu. J Infect Dis 184: 1163-1169.

Yadav KK, Adhikari N, Khadka R, Pant AD and Shah B (2015). Multidrug resistant Enterobacteriaceae and extended spectrum beta-lactamase producing Escherichia coli: a cross-sectional study in National Kidney Center, Nepal. Antimicrob Resist Infect Control 4:42. 